\title{
Ddx41 inhibition of DNA damage signaling permits erythroid progenitor expansion in zebrafish
}

Haematologica 2022

Volume 107(3):644-654

\section{Joshua T. Weinreb, ${ }^{1,2}$ Varun Gupta, ${ }^{3}$ Elianna Sharvit, ${ }^{1}$ Rachel Weil, ${ }^{1}$ and Teresa V. Bowman ${ }^{1,2,4}$}

${ }^{1}$ Albert Einstein College of Medicine, Department of Developmental and Molecular Biology; ${ }^{2}$ Albert Einstein College of Medicine, Gottesman Institute for Stem Cell Biology and Regenerative Medicine; ${ }^{3}$ Albert Einstein College of Medicine, Department of Cell Biology and ${ }^{4}$ Albert Einstein College of Medicine and Montefiore Medical Center, Department of Medicine (Oncology), Bronx, NY, USA

\section{ABSTRACT}

D EAD-box Helicase 41 (DDX41) is a recently identified factor mutated in hematologic malignancies whose function in hematopoiesis is unknown. Using an in vivo model of $\mathrm{Ddx} 41$ deficiency, we unveiled a critical role for this helicase in regulating erythropoiesis. We demonstrated that loss of $d d \times 41$ leads to anemia caused by diminished proliferation and defective differentiation of erythroid progenitors. Mis-expression and alternative splicing of cell cycle genes is rampant in $d d \times 41$ mutant erythroid progenitors. We delineated that the DNA damage response is activated in mutant cells resulting in an Ataxiatelangiectasia mutated (ATM) and Ataxia-telangiectasia and Rad3-related (ATR)-triggered cell cycle arrest. Inhibition of these kinases partially suppressed $d d \times 41$ mutant anemia. These findings establish a critical function for $\mathrm{Ddx} 41$ in promoting healthy erythropoiesis via protection from genomic stress and delineate a mechanistic framework to explore a role for ATM and ATR signaling in DDX41-mutant hematopoietic pathologies.

\section{Introduction}

Mutations in DEAD-box Helicase 41 (DDX41) were identified recently in hematologic malignancies including myelodysplastic syndrome (MDS), acute myeloid leukemia (AML), and acute erythroid leukemia (AEL).,2 Germline DDX41 frameshift mutations are loss-of-function and somatic missense mutations are thought to result in hypomorphic helicase activity. ${ }^{1,3}$ The human genetics thus suggest that diminished function of this helicase is detrimental to hematopoiesis, but this has yet to be demonstrated in an animal model.

In particular, a significant number of DDX41-mutated MDS patients experience mild cytopenia in the years preceding diagnosis, indicating that anemia may be one of the first warning signs of disease. ${ }^{4}$ Anemia in MDS is attributed to numerous cellular mechanisms including erythroid precursor apoptosis, defective progenitor expansion, and ineffective erythrocytic maturation. ${ }^{5.7}$ The clinical findings suggest DDX41 could be important in erythropoiesis, but the cellular and molecular underpinnings remain unclear. Roles for DDX41 have been implicated in genomic stability, inflammation, and splicing, all processes linked to hematopoietic health, but the current lack of DDX41 mutant animal models has slowed exploration of its function in the blood system. ${ }^{8 \cdot 11}$

In order to uncover the in vivo role of DDX41 in erythropoiesis, we established a zebrafish $d d \times 41$ loss-of-function mutant. We demonstrated that $d d \times 41$ mutants develop anemia due to a decrease in erythroid progenitor expansion and defective differentiation. Mechanistically, the erythroid proliferative defect is due in part to ATM- and ATR-mediated cell cycle arrest induced by elevated DNA damage as well as mis-expression and alternative splicing of cell cycle regulators. Our data demonstrate that Ddx41 plays a critical role in hematopoiesis and provide a possible mechanism by which anemia may arise in DDX41-mutated hematopoietic pathologies. 


\section{Methods}

\section{Zebrafish}

Zebrafish were maintained as described ${ }^{12}$ and according to Institutional Animal Care and Use Committee-approved protocols in accordance with the Albert Einstein College of Medicine research guidelines. Genotyping was performed to confirm animal identity. Mutants for $d d x 41\left(d d \times 41^{\text {san } 1488}\right)$ were acquired from the Zebrafish International Resource Center. ${ }^{13}$ The mutation results in a premature stop codon at tyrosine 410 . For all experiments, sibling controls are a mix of heterozygotes and wild types. $\operatorname{Tg}(\text { gata1:dsred })^{14}$ transgenics were used. Genotyping details are in the Online Supplementary Appendix and the Online Supplementary Table S7.

\section{Drug treatments}

All drugs were dissolved in dimethyl sulfoxide (DMSO). Dilutions were made in E3 embryo water. KU60019 (ATM inhibitor) and AZ20 (ATR inhibitor) were used with DMSO as the vehicle control.

\section{Whole-mount in situ hybridization and 0-dianisidine staining}

In situ hybridization was performed as previously described. ${ }^{15,16}$ After in situ, embryos were scored manually, imaged and genotyped. The $\beta e 3$-globin, ${ }^{17} \mathrm{cmyb},{ }^{18}$ and gata ${ }^{19}$ probes were used, and in situ levels were quantified using FIJI. ${ }^{20}$ O-dianisidine staining was performed as previously described. ${ }^{21}$

\section{Flow cytometry}

Mutant and sibling embryos were binned based on morphological differences. For generation of single-cell suspensions, 1020 embryos were processed as previously described ${ }^{15}$ (also see the Online Supplementary Appendix). Quantification for the absolute number of cells was performed by acquiring all events in a tube on the flow cytometer to determine the total number of target cells. This number was then divided by the total number of embryos analyzed to calculate the number of target cells per embryo.

\section{Cell cycle and apoptosis analyses}

For 5-ethynyl-2'-deoxyuridine (EdU) incorporation experiments, embryos were incubated with $20 \mathrm{mM}$ EdU for 2 hours. Single-cell suspensions of embryos were generated. Click-IT EdU Flow Cytometry Assay Kit was used according to the manufacturer's instructions. Flow cytometry analysis for active caspase- 3 was performed as previously described. ${ }^{22}$ Samples were analyzed with a LSRII flow cytometer (BD Biosciences) and FlowJo software.

\section{RNA sequencing and splicing analysis}

Erythroid progenitors from $d d x 41$ mutants and siblings were isolated by fluorescently-activated cell sorting (FACS). RNA from these cells was subsequently isolated, DNAse-digested and library prepared for sequencing. Details on library preparation, sequencing and bioinformatic analyses can be found in the Online Supplementary Appendix. All data are deposited under GEO accession number GSE160979.

\section{Reverse transcription quantitative polymerase chain reaction}

In order to validate the RNA sequencing (RNA-seq) data, we performed reverse transcription quantitative polymerase chain reaction (RT-qPCR). RNA was isolated from $40 \mathrm{hpf}$ embryos. Details are listed in the Online Supplementary Appendix and Online Supplementary Table S7.

\section{Single-cell immunofluorescence of zebrafish embryonic cells}

Single-cell suspensions were prepared, and cell staining was performed as described in Sorrells \& Nik et al. ${ }^{22}$ and detailed in the Online Supplementary Appendix. Fluorescence intensity measurements of $\gamma \mathrm{H} 2 \mathrm{AX}$ were performed using FIJI.

\section{May-Grunwald Giemsa staining of primitive erythroid cells}

May-Grunwald Giemsa staining was performed as previously described ${ }^{15}$ and as detailed in the Online Supplementary Appendix.

\section{Statistics}

Experiments were performed with a minimum of three replicates. Statistical analyses were performed as indicated in each figure using unpaired Student's $t$-test or a one-way ANOVA with Tukey's multiple testing correction as appropriate; error bars indicate the standard deviation of mean, unless otherwise indicated.

\section{Results}

\section{Ddx41 regulates erythroid development}

In order to explore a function for Ddx41 in hematopoiesis, we examined erythrocyte formation and differentiation in zebrafish $d d \times 41$ homozygous loss-offunction mutants $\left(d d \times 41^{\text {sal } 4887}\right.$. Maternally-deposited Ddx41 (data not shown) helps the mutants develop and survive until 3 days post fertilization (dpf) (Online Supplementary Figure $S 1 A$ and $B$ ). Based on this, we consider the mutants to be functionally hypomorphic with greatly diminished but not completely absent Ddx41 levels. DDX41 is highly conserved between humans and zebrafish suggesting that lessons learned about the in vivo role of zebrafish Ddx41 function in hematopoiesis will be relevant to human DDX41. In zebrafish, primitive hematopoiesis begins $\sim 12-24$ hours post fertilization (hpf), producing embryonic erythrocytes and myeloid cells that constitute the hematopoietic system early on in development. ${ }^{23,24}$ Cells of the erythrocytic lineage first arise from the intermediate cell mass (ICM) within the posterior lateral mesoderm (PLM) (Figure 1A). These erythrocytes express factors such as the progenitor transcription factor c-myb and the erythroid-specific transcription factor gata1 starting during somitogenesis. ${ }^{19,25}$ Using in situ hybridization, we determined that these erythroid progenitor markers were expressed similarly in $d d x 41$ mutants compared to siblings (mix of $d d x 41$ heterozygotes + wild types) at $22 \mathrm{hpf}$, indicating initial erythroid specification is unaffected (Figures 1B to E). Oxygenated hemoglobinized erythrocytes are detectable beginning around $36 \mathrm{hpf}$ using o-dianisidine staining. ${ }^{26}$ In $d d \times 41$ mutants, we observed little o-dianisidine-positive erythroid cells at $40 \mathrm{hpf}$ (Figure 1F). We sorted gata1:dsRed erythrocytes at $40 \mathrm{hpf}$ and found that the $d d \times 41$ mutant cells were larger than those from sibling controls (Figure 1G). This size difference could be indicative of delayed erythroid differentiation. As mutants display some developmental delay that becomes more severe as the embryos get older, it is possible that the erythroid delay is a side effect of the general developmental delay. In order to distinguish between these possibilities, we examined erythrocytes in $d d x 41$ mutants and siblings at $48 \mathrm{hpf}$. Oxygenated hemoglobin levels remained low in mutants at $48 \mathrm{hpf}$ (Figures $1 \mathrm{H}$ to I). In order to assess maturation, we also bled $d d \times 41$ mutants and sibling control embryos at $48 \mathrm{hpf}$ and analyzed the morphology of isolated 


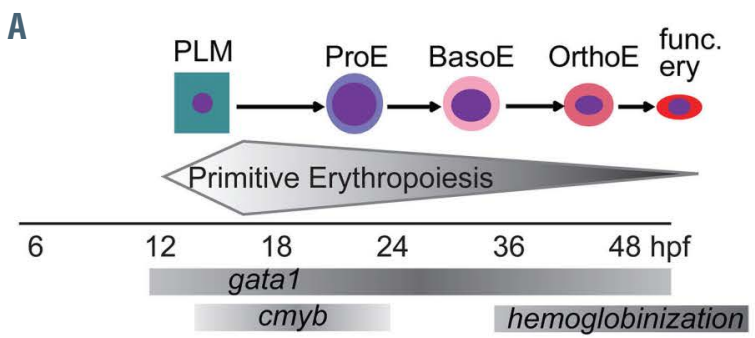

B

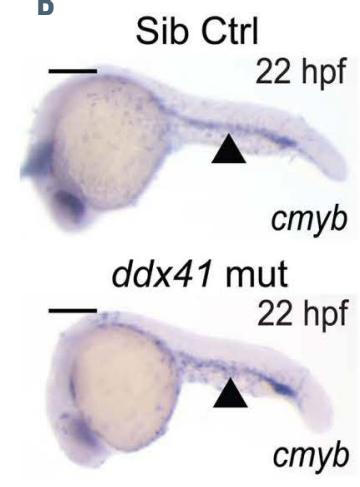

C

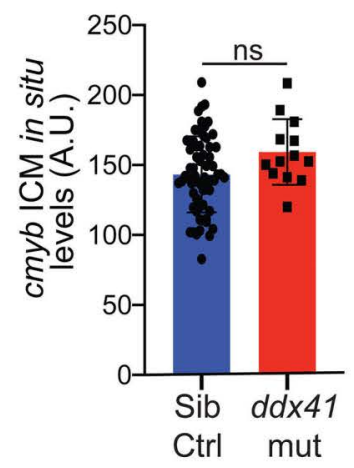

D

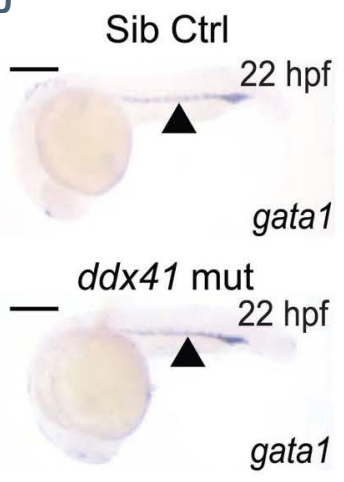

E

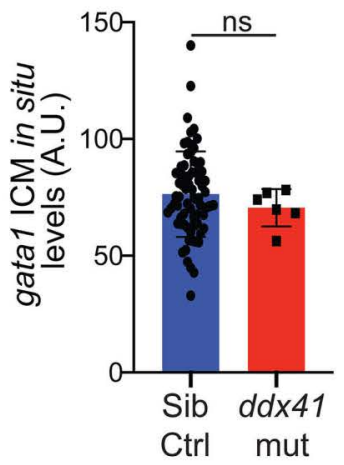

$\mathrm{F}$

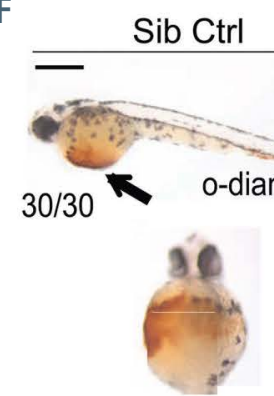

H
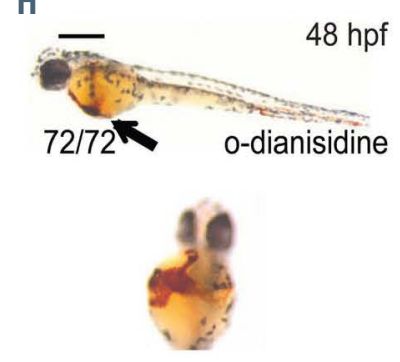

J

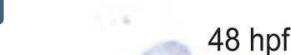

$168 / 190$

$88.4 \%$
$48 \mathrm{hpf}$ $d d \times 41$ mut
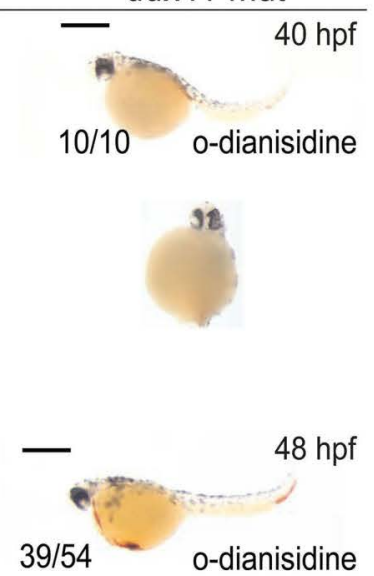

$118 / 144$

$81.9 \%$
G

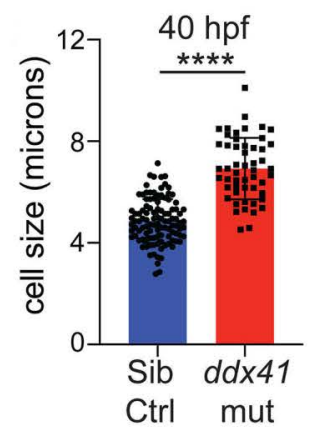

Figure 1. Loss of $d d x 41$ causes anemia. (A) Schema of primitive erythroid development, PLM: posterior lateral mesoderm; ProE: proerythroblasts; BasoE: basophilic erythroblasts; OrthoE: orthochromatophilic erythroblasts; func. Ery: functional erythrocytes. (B and D) In situ hybridization of the erythroid markers cmyb (B) (scale bars $=200$ $\mu \mathrm{m}$ ) and gata1 (D) (scale bars $=250 \mu \mathrm{m}]$ at 22 hours post fertilization (hpf) in sibling controls (top) and ddx41 mutants (bottom). Arrowheads highlight the intermediate cell mass (ICM) region in the embryos. (C and E) Quantification of c-myb (C) and gata1 (E) in situ hybridization levels from (B) and (D), respectively. Quantification was done using Fiji. ( $\mathrm{F}$ and $\mathrm{H}$ ) Staining for o-dianisidine, marking functional hemoglobin in mature primitive erythrocytes, in sibling controls (left) and ddx41 mutants (right) at $40 \mathrm{hpf}(\mathrm{F})(\mathrm{scale}$ bars $=350 \mu \mathrm{m})$ and $48 \mathrm{hpf}(\mathrm{H})$ (scale bars $=400 \mu \mathrm{m})$. Numbers on bottom left corner indicate the fraction of embryos with the same phenotype as the one depicted in the image. (G) Graph depicting size of erythrocytes in sibling controls and ddx41 mutants at 40 hpf. (I) Graph depicting frequency of designated odianisidine staining levels in sibling controls and ddx41 mutants at $48 \mathrm{hpf}$. (J) Representative images of orthochromatophilic erythroblasts stained with May-GrunwaldGiemsa from sibling controls (left) and $d d x 41$ mutants (right) at $48 \mathrm{hpf}$ (scale bars $=5 \mu \mathrm{m}$ ). Graphs display means \pm standard deviations (stds) with $P$-values calculated with unpaired Student's $t$-test, ns $=$ not significant $(P>0.05)$, $* * * * P>0.0001$. For in situs and o-dianisidine staining $n=6-72$ embryos per experiment. 
erythrocytes using May-Grunwald-Giemsa staining (Figure 1J). The $d d \times 41$ mutant erythrocytes displayed a megaloblastoid-like phenotype, suggesting some abnormalities in erythrocyte maturation.

In order to acquire enough erythrocytes to perform the morphological assessment, we needed to bleed four times as many $d d \times 41$ mutant embryos as compared to sibling control embryos, suggesting mutants had fewer erythrocytes than siblings. In order to test this hypothesis, we assessed the number of gata1:dsred ${ }^{+}$erythroid progenitors in $d d \times 41$ mutants and siblings using flow cytometry quantification. We determined that the absolute number of gata1:dsred ${ }^{+}$erythrocytes per embryo was significantly reduced in $d d \times 41$ mutants compared to siblings at both 28 and $40 \mathrm{hpf}$ (Figures $2 \mathrm{~A}$ to $\mathrm{D}$ ). These data indicate that decreased erythrocyte number contributes to the development of anemia in $d d \times 41$ mutants.

Erythroid progenitors arising from both primitive and definitive erythroid-myeloid progenitor (EMP)-derived waves are present during the developmental time points analyzed. The gene programs for the specification and differentiation of primitive and EMP-derived erythropoiesis are highly similar, but the developmental timings are distinct (Figure 2E). EMP specification begins around 26 hpf. $^{27}$ In order to determine whether there were defects in EMPderived erythropoiesis, we performed in situ hybridization for the progenitor marker c-myb at $26 \mathrm{hpf}$ and $36 \mathrm{hpf}$ and gata1 at $26 \mathrm{hpf}$ in siblings and $d d \times 41$ mutants (Figures $2 \mathrm{~F}$ and G; Online Supplementary Figure S1C to F). Expression of both $c-m y b$ and gata 1 within the posterior blood island (PBI) region where EMP form were not decreased and in fact were increased in $d d \times 41$ mutants as compared to siblings. As the gene programs are highly similar between these two waves of erythroid development, these data indicate that the reduction in erythrocytes in $d d \times 41$ mutants is occurring at an erythroid progenitor stage after $c-m y b$ and gata1 are both expressed, which is shortly after erythroid lineage specification.

In order to further characterize the maturation state of the erythrocytes at $40 \mathrm{hpf}$ in $d d \times 41$ mutants and siblings, we performed RT-qPCR for embryonic and larval globins. Expression of the embryonic globins $\alpha \varepsilon 1, \alpha \varepsilon 3, \beta \varepsilon 1$, and $\beta \varepsilon 3$ begins during somitogenesis with expression of all of these globins except $\beta \varepsilon 3$ persisting in primitive and EMP-derived erythrocytes throughout larval development. ${ }^{28}$ In contrast, levels of $\beta \varepsilon 3$ globin diminish dramatically from $24-48 \mathrm{hpf}$, somewhat concomitant with the increasing expression of the larval $\beta \varepsilon 2$ globin. The other larval globin $\alpha \varepsilon 5$ is not expressed significantly until $14 \mathrm{dpf}$. In $d d \times 41$ mutants, we determined that while the levels of the embryonic/larval globins $\alpha \varepsilon 1$ and $\beta \varepsilon 1$ were diminished, the levels of the embryonic-restricted $\beta \varepsilon 3$ globin remained high, consistent with a maturational defect in primitive erythrocytes. Additionally, expression of the larval $\beta \varepsilon 2$ globin was lower in mutants compared to sibling controls. Although $d d \times 41$ mutants die before there are expansive numbers of maturing erythrocytes derived from EMP, these data indicate that mutants have fewer definitive erythrocytes compared to siblings. This finding suggests that similar to primitive erythroid progenitors, EMP are specified normally, but there is a later stage defect, although the underlying cause (e.g., diminished expansion, maturation or differentiation) cannot be deciphered. Together, our findings establish that $\mathrm{Ddx} 41$ is critical for erythrocyte expansion and maturation.

\section{Cell cycle genes are mis-expressed and alternatively} spliced in ddx41 mutant erythroid progenitors

In order to mechanistically assess the underlying cause of the erythrocytic defect in $d d x 41$ mutants, we conducted RNA-seq on gata1:dsred ${ }^{+}$erythrocytes isolated from $d d \times 41$ mutants and siblings at $40 \mathrm{hpf}$. Over 1,800 genes were downregulated and more than 1,900 were upregulated in ddx41 mutants compared to siblings (Figure 3A; Online Supplementary Table $S 1$, log fold-change $\geq 1$, adjusted $P$ value $<0.05$ ). In order to understand if particular pathways were enriched in the differentially expressed genes, we performed gene set level analysis on the upregulated and downregulated gene lists by comparing each to the Molecular Signature Database (MSigDb), a platform that computes overlaps between classes of genes that are overor underrepresented in lists of genes in known pathways. ${ }^{29,30}$ In the downregulated gene list, mRNA splicing was the top gene set with DNA replication, cell cycle, and DNA repair also enriched (Figure 3B; Online Supplementary Table S2). In the upregulated gene list, genes associated with adaptive immunity, posttranslational modifications, innate immune system, and cell cycle were enriched (Figure 3C; Online Supplementary Table S3). We validated the expression changes in several cell cycle and DNA-damage-associated genes using RT-qPCR (Figure 3D).

Ddx41 interacts with components of the spliceosome. ${ }^{1}$ Additionally, the top downregulated pathway in our gene set was pre-mRNA splicing, thus we examined how $d d \times 41$ loss affected mRNA splicing in erythrocytes. When comparing splicing between $d d \times 41$ mutants and siblings, a total of 370 alternative splicing events were observed (Figures 3E; Online Supplementary Table S4). The specific splicing defects detected included exon skipping (SE), which was the most frequently altered splicing event, intron retention (RI), alternative 5 '-splice site usage, alternative 3 '-splice site usage, and changes in mutually exclusive exon usage. Alternative splicing within protein coding regions of a transcript can result in the introduction of premature termination codon (PTC) or generation of a novel peptide. For all SE and RI events (comprising nearly $85 \%$ of all splicing changes), we determined how the alternative splicing event might alter the protein sequence (Figure 3F; Online Supplementary Table S5). More than $50 \%$ of SE events altered the protein sequence and are predicted to generate novel peptides. Approximately $43 \%$ of SE and $90 \%$ of RI events are predicted to target the alternatively spliced transcript for nonsense-mediated decay (NMD) due to the introduction of a PTC. For example, the retained intron variant for homologous repair-associated factor structural maintenance of chromosome 5 (smc5) identified in $d d \times 41$ mutants is predicted to result in NMD that could result in elevated DNA damage (Figure $3 \mathrm{G}$ ). Another example of an NMD isoform expressed in $d d \times 41$ mutant is the exon 3 skipped isoform of signal transducer and activator of transcription 1a (stat1a) that would diminish signaling by numerous cytokine pathways. Pathway analysis of these alternatively spliced factors revealed that those resulting in novel peptide sequences are enriched in mRNA metabolism, morphogenesis, and cell cycle, and those predicted to result in NMD are enriched for mRNA processing, DNA replication, and gene expression (Figure 3H; Online Supplementary Table S6). These results depict that Ddx41 influences the expression and splicing of cell cycle, DNA repair, and mRNA processing genes in erythrocytes. 


\section{Ddx41 deficiency triggers cell cycle arrest in erythroid} progenitors

The diminished number of erythroid progenitors and dysregulated expression of cell cycle genes suggest that defects in erythrocyte proliferation could contribute to the anemia in $d d x 41$ mutants. In order to examine proliferation, we analyzed cell cycle status of $30 \mathrm{hpf}$ gata1:dsred ${ }^{+}$erythroid progenitors by flow cytometry quantification of DNA synthesis via EdU incorporation and DNA content via DAPI incorporation. The $d d \times 41$

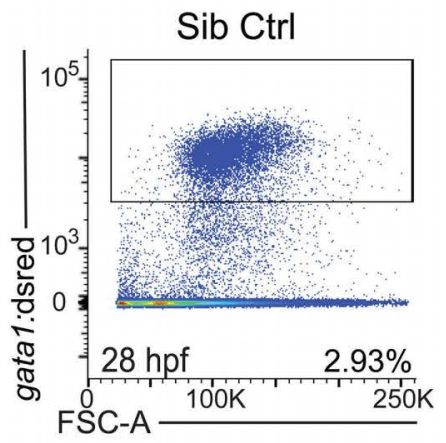

C

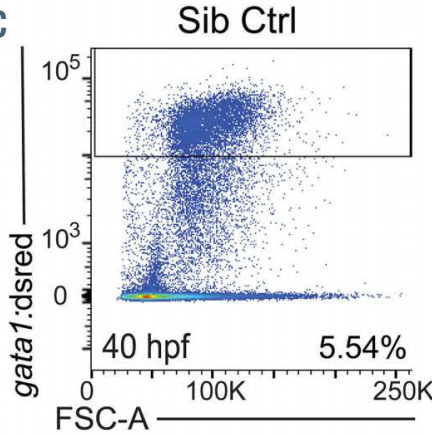

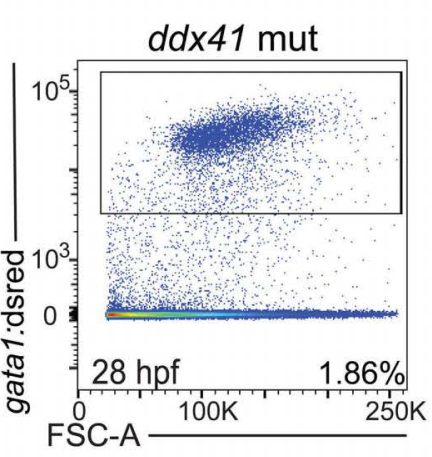

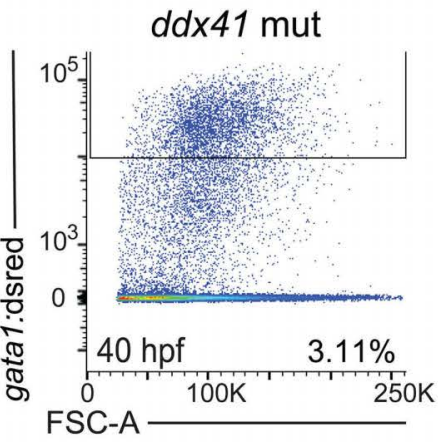

B

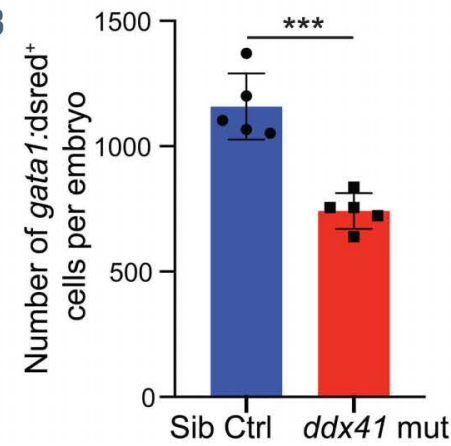

D

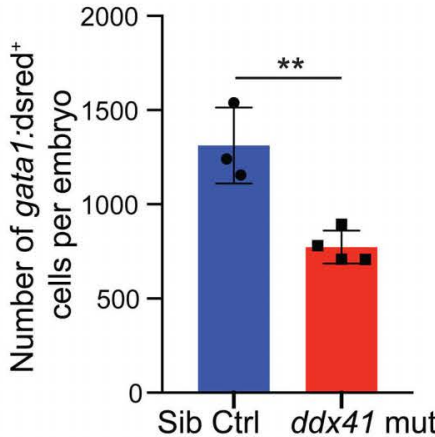

E EMP ProE BasoE OrthoE func

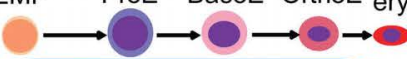

EMP-derived Erythropoiesis

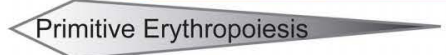

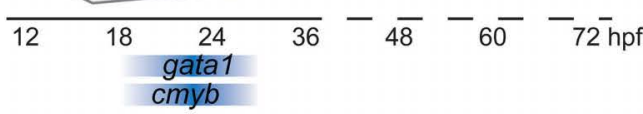

F

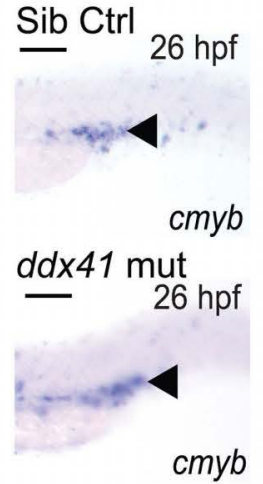

G

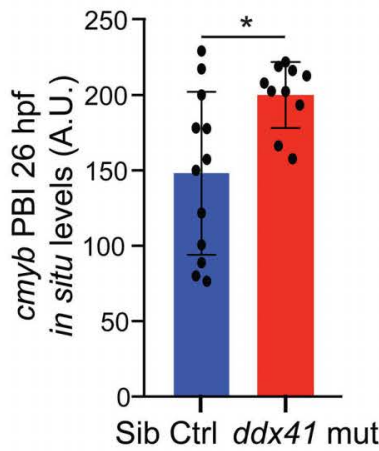

H

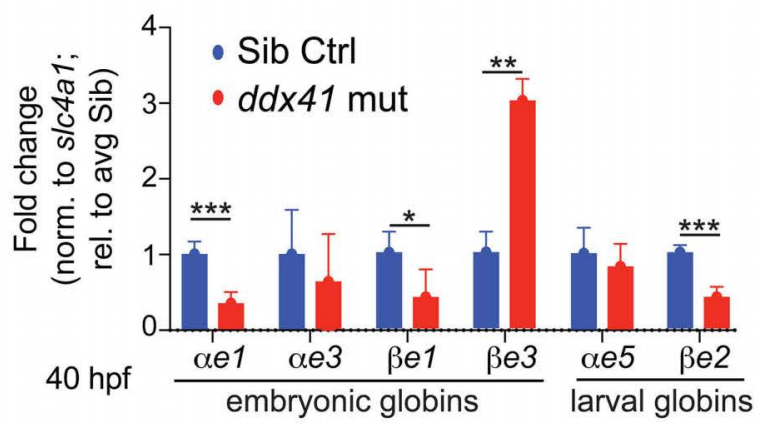

Figure 2. Ddx41 regulates erythroid progenitor numbers. (A and C) Flow cytometry plots of gata1:dsred ${ }^{+}$erythroid cells from sibling controls (left) and ddx41 mutants (right) at 28 days post fertilization (dpf) (A) and $40 \mathrm{hpf}(\mathrm{C})$. (B and D) Graphs depicting the absolute number of gata1:dsred+ erythroid cells per embryo from (A) and (C), respectively. $n=5$ pools of $\sim 5-20$ embryos per pool. (E) Schema of erythroid-myeloid progenitor (EMP) development. ProE: proerythroblasts; BasoE: basophilic erythroblasts; OrthoE: orthochromatophilic erythroblasts; func. Ery: functional erythrocytes. (F) In situ hybridization of $\mathrm{cmyb}$ at $26 \mathrm{hpf}$ in sibling controls (left) and ddx41 mutants (right) (scale bars $=150 \mu \mathrm{m}$ ). (G) Quantification of cmyb PBI in situ hybridization levels from (F). Quantification was done using Fiji; $\mathrm{n}=10-12$ embryos. Graphs display means \pm standard deviations (stds). (H) Graph of reverse transcription quantitative polymerase chain reaction (RT-qPCR) analysis of the expression of globin genes between sibling controls and $d d x 41$ mutants. Expression levels were normalized to s/c $4 a 1$ levels. Graph displays means \pm standard error mean. The $P$-values were calculated with an unpaired $t$-test, $* P<0.05, * * P \leq 0.01, * * * P \leq 0.001 ; n=3$ replicates per genotype. 
A

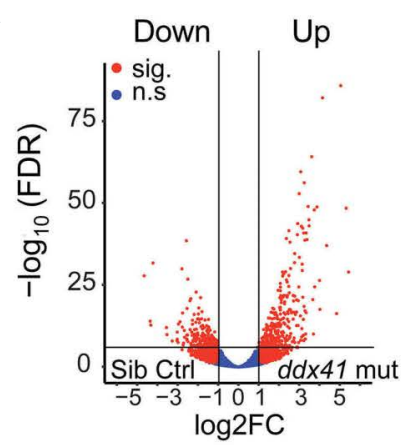

B

Pathway analysis:

Downregulated genes

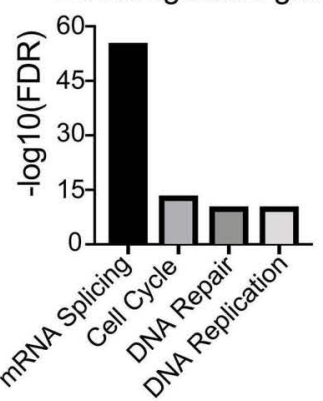

C Pathway analysis:

Upregulated genes
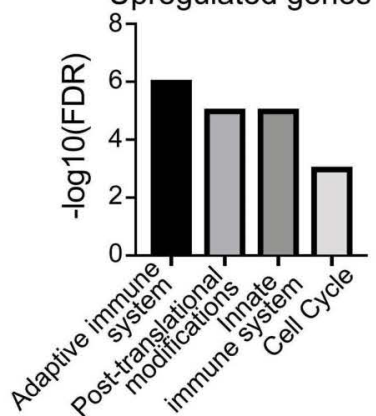

D

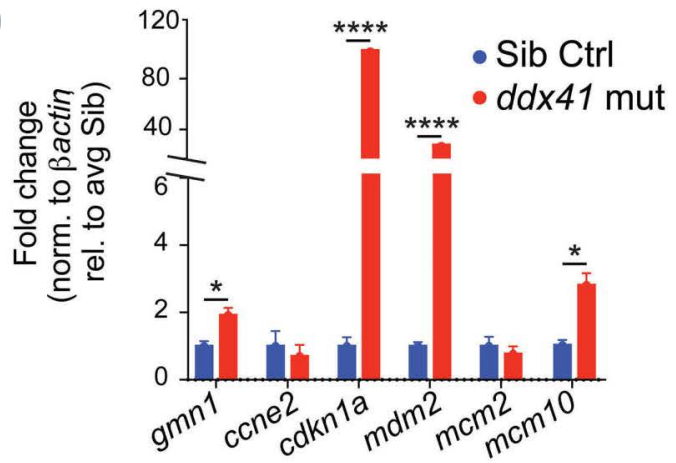

E

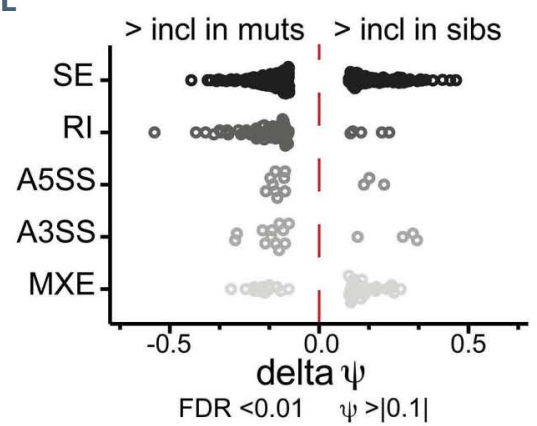

F

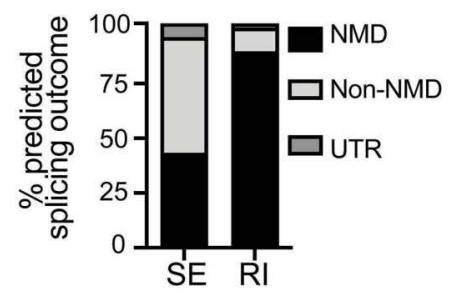

G
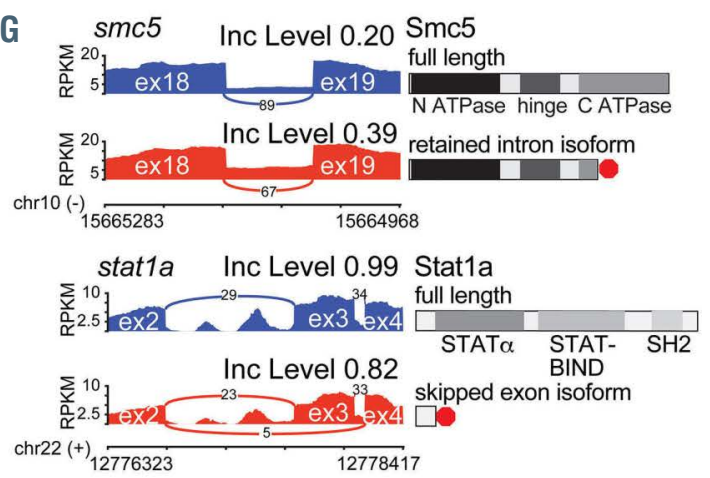

H Pathway analysis:

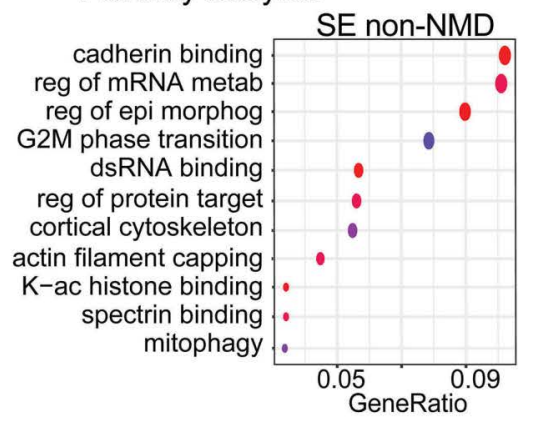
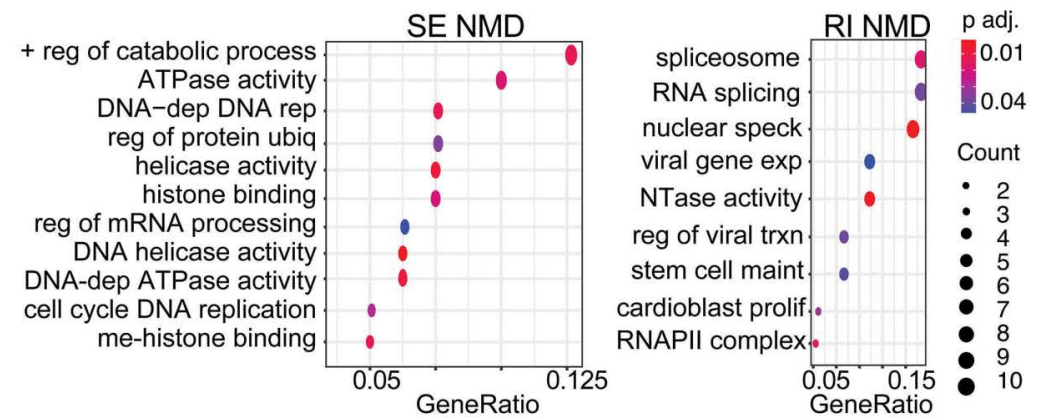

Figure 3. Cell cycle genes are mis-expressed and alternatively spliced in $d d x 41$ mutant erythroid progenitors. (A) Volcano plot displaying differentially expressed genes between gata1:dsred ${ }^{+}$erythrocytes from $d d x 41$ mutants and siblings. Significant differences are defined as false discovery rate (FDR) $<0.05$ and log fold-change $\geq 1$. Black vertical lines denote the fold-change threshold and the black horizontal line denotes the FDR threshold. Five biological replicates for both $d d x 41$ mutants and siblings were used to generate RNA sequencing data. (B and C). Representative charts of pathways significantly enriched in genes downregulated (B) or upregulated (C) in ddx41 mutant erythroid progenitors compared to sibling controls as determined by MSigDB analysis. (D) Graph of reverse transcription quantitative polymerase chain reaction (RT-qPCR) analysis of the expression of cell cycle and DNA damage-associated genes between sibling controls and ddx41 mutants. Expression levels were normalized to $\beta$-actin levels. Graph displays means \pm standard error mean. The $P$-values were calculated with an unpaired $t$-test, $* P<0.05, * * * * P \leq 0.0001$; $n=3$ replicates per experiment. (E) Graph depicting the $\Delta \psi$ of individual splicing events between sibling controls and $d d \times 41$ mutants as detected by analysis with rMATS. Significant differences are defined as FDR $\leq 0.01$ and $\Delta \psi \geq 0.1$. SE: skipped exons; RI: retained introns; A5SS: alternative 5' splice site; A3SS: alternative 3' splice site; MXE: mutually exclusive exons. (F) Graph depicting the frequency of alternatively spliced isoforms in $d d x 41$ mutants that are predicted to result in nonsense-mediated (NMD) decay, protein sequence alterations (nonNMD), or changes in untranslated regions (UTR). (G) Sashimi plot for smc5 (exons 18-19) and stat1a (exons 2-4) in ddx41 mutant erythrocytes compared to sibling controls. RPKM: reads per kilobase of transcript per million mapped reads; Inc: inclusion. (H) Representative charts of pathways significantly enriched in alternatively spliced genes in $d d \times 41$ mutant erythrocytes compared to sibling controls as determined by pathway analysis. 
mutant gata1:dsred ${ }^{+}$progenitors showed a reduction of cells in $\mathrm{S}$ phase and an accumulation of cells in the G0/G1 and G2/M phases compared to sibling controls (Figures $4 \mathrm{~A}$ and $\mathrm{B}$ ). These results are in-line with a decrease in proliferation in $d d \times 41$ mutant erythrocytes caused by cell cycle arrests at the G0/G1-to-S phase and G2-to-M transitions. Prolonged cell cycle arrest can lead to apoptosis, ${ }^{31}$ thus we also assessed apoptosis in $d d \times 41$ mutants. We measured levels of active caspase- 3 , an essential executor of apoptosis, in $d d \times 41$ mutant and sibling gata1:dsred ${ }^{+}$erythrocytes by flow cytometry. We observed a significant increase in active caspase- 3 in ddx41 mutant gata1:dsred ${ }^{+}$erythrocytes at $30 \mathrm{hpf}$ (Figures 4C-D). These data indicate that both cell cycle arrest and elevated apoptosis in $d d \times 41$ mutant erythrocytes may drive anemia.

\section{Ddx41 regulation of ATM and ATR signaling contributes to proper erythropoiesis}

These molecular and cellular phenotypes in $d d \times 41$ mutants imply that loss of $d d \times 41$ could promote DNA damage. In order to address this question, we analyzed the DNA damage response (DDR) by performing immunofluorescence (IF) for $\gamma \mathrm{H} 2 \mathrm{AX}$ in $d d \times 41$ mutants and siblings. We showed that $\gamma \mathrm{H} 2 \mathrm{AX}$ levels were increased nearly two-fold in $d d \times 41$ mutants compared to sibling cells (Figures $4 \mathrm{E}$ and F). These data demonstrate that $d d \times 41$ deficiency triggers DDR in vivo.
A
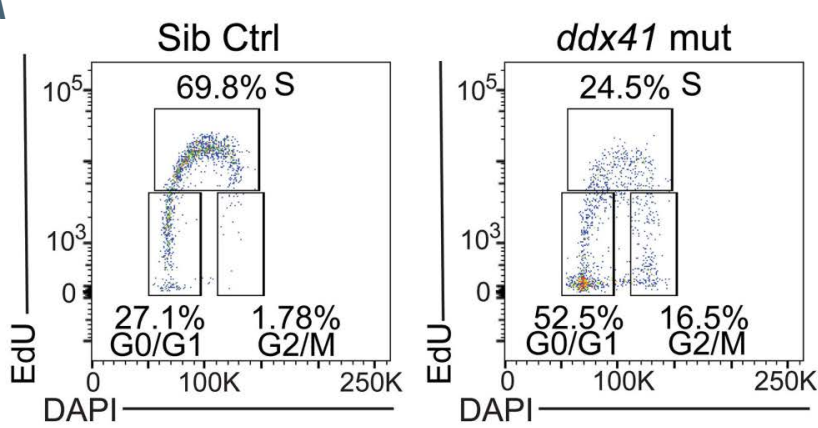

C
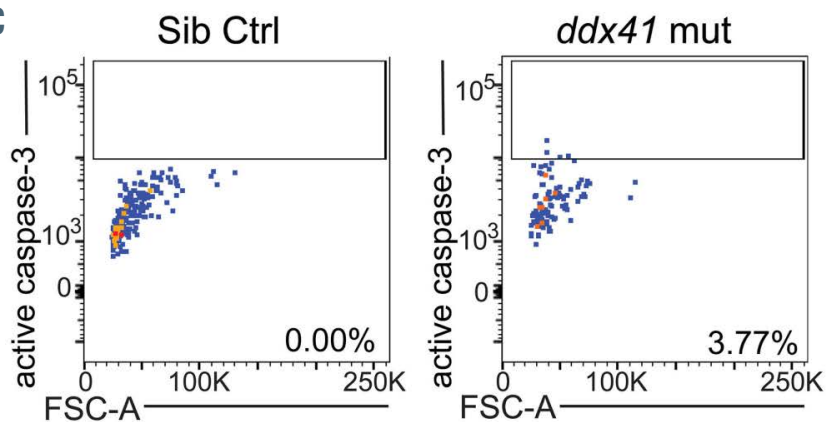

E
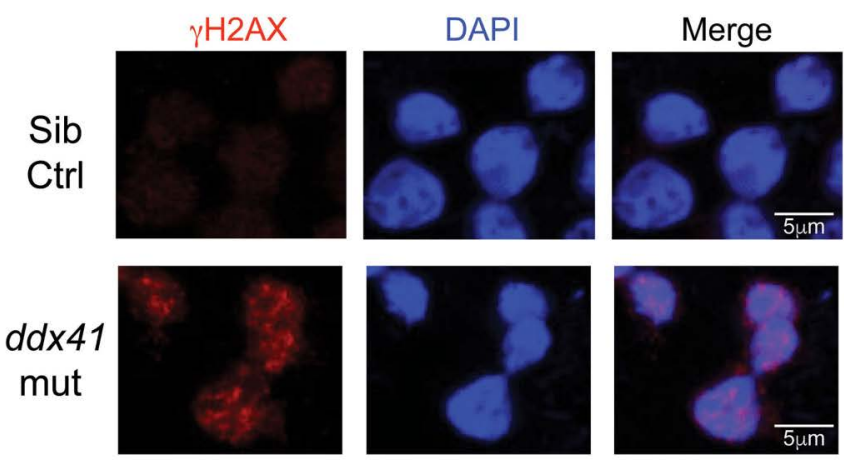
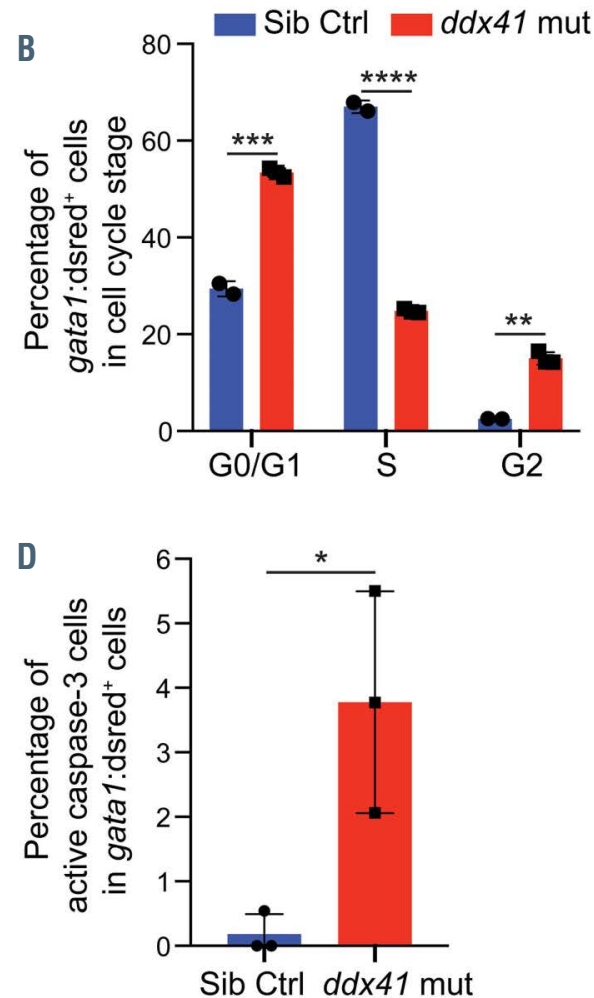

F

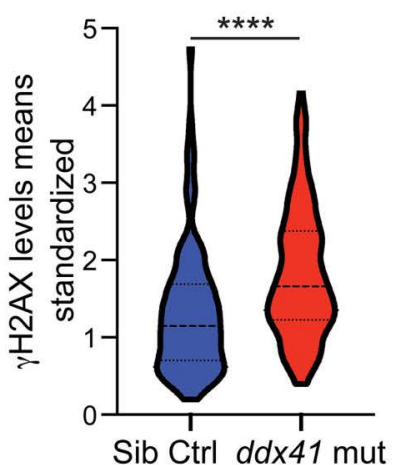

Figure 4. Ddx41 deficiency triggers cell cycle arrest and DNA damage response in erythroid progenitors. (A) Cell cycle analysis of gata1:dsred ${ }^{+}$erythroid cells from sibling controls (left) and ddx41 mutants (right) after a 2-hour pulse of 5-ethynyl-2'-deoxyuridine (EdU) at 28 hpf. EdU incorporation ( $y$-axis) and DAPI content (x-axis) were measured by flow cytometry at 30 hours post fertilization (hpf). (B) Quantification of the percentage of cells in each cell cycle phase from (A). (C) Flow cytometry analysis of active-caspase 3 in gata1:dsred ${ }^{+}$erythroid cells from sibling controls (left) and ddx41 mutants (right). (D) Quantification of the percentage of gata1:dsred ${ }^{+}$ erythroid cells that are active caspase-3-positive from (C). (E) Confocal images showing immunofluorescence of nuclei (DAPI) and $\gamma \mathrm{H} 2 \mathrm{AX}$ in cells isolated from $28 \mathrm{hpf}$ siblings (top) and $d d \times 41$ mutants (bottom). scale bars $=5 \mu \mathrm{m}$. (F) Quantification of $\mathrm{YH} 2 \mathrm{AX}$ levels from (E). Graphs display means \pm standard deviations (stds) with $P$ values calculated with unpaired Student's $t$-test, $* P<0.05, * * P \leq 0.01, * * * P \leq 0.001, * * * * P \leq 0.0001$. For flow cytometry, $\mathrm{n}=3-5$ pools of $\sim 5-20$ embryos per pool. For immunofluorescence imaging, $n=100-300$ cells per genotype. 
Our model is that loss of Ddx41 contributes to excessive DDR signaling and subsequent cell cycle arrest in erythrocytes, leading to anemia in $d d \times 41$ mutants. If correct, then inhibiting components of the DDR pathway would i) reverse cell cycle defects and ii) increase erythrocyte levels. In order to test this model, we examined how the two primary mediators of DDR, Ataxia-telengiectasia-mutated (ATM) and Ataxia-telengiectasia and Rad3-related (ATR), affected erythrocytic cell cycle kinetics in $d d \times 41$ mutants. We assessed cell cycle status of $30 \mathrm{hpf} d d \times 41$-mutant gata1:dsred ${ }^{+}$erythroid progenitors in embryos treated with DMSO vehicle control, the ATM inhibitor KU60019, or the ATR inhibitor AZ20. There was a significant increase of gata1:dsred ${ }^{+} d d \times 41$-mutant cells in $\mathrm{S}$ phase when treated with either ATM or ATR inhibitors as compared to DMSO vehicle control (Figures $5 \mathrm{~A}$ and $\mathrm{B}$ ). Additionally, pharmacological inhibition of ATM or ATR increased erythropoietic output in $d d \times 41$ mutants, as measured by quantification of gata1:dsred ${ }^{+}$erythrocyte numbers per embryo using flow cytometry (Figures 5C to F). Although there was a trend towards an increase in erythrocyte numbers in control siblings treated with ATM or ATR inhibitors these changes were not statistically significant. Taken together, these data indicate that DDR signaling triggers a G0/G1 cell cycle arrest in $\mathrm{ddx} 41$-mutant erythrocytes that results in a reduction of erythroid progenitor cell number.

Finally, we wanted to assess if increasing the number of erythroid progenitors via ATM or ATR inhibition would increase the number of oxygenated erythrocytes in $d d \times 41$ mutants. Surprisingly, we only observed a significant increase in o-dianisidine-positive erythrocytes in $d d \times 41$ mutants treated with ATM inhibitor, but not ATR inhibitor (Figures 6A and B). These data indicate that Ddx41 regulation of ATM might have a broader impact on erythropoiesis than ATR signaling.

\section{Discussion}

Although DDX41 mutations are found in numerous human hematologic diseases, its function in hematopoiesis is unknown. Our work is the first to establish Ddx41 as a critical mediator of erythropoiesis with $d d \times 41$ loss suppressing the expansion and maturation of erythrocytes. We showed a profound effect on the expression of cell cycle and DNA damage-associated genes in $d d \times 41$ mutant erythroid progenitors consistent with the observed cell cycle arrest. The DNA damage response is elevated in ddx41 mutant cells and triggers an ATM and ATR-triggered cell cycle arrest. Inhibition of ATM and ATR partially suppressed anemia in $d d \times 41$ mutants. These findings establish $\mathrm{Ddx} 41$ as a positive regulator of erythropoiesis in part by preventing genomic stress and promoting proper erythroid progenitor expansion.

Patients with germline mutations in DDX41 do not develop hematologic symptoms until later in life, ${ }^{1}$ yet zebrafish ddx41 mutants show anemia within $40 \mathrm{hpf}$. We posit that the difference has to do with the extent of Ddx41 deficiency. Zebrafish homozygous mutants have maternally deposited $\mathrm{Ddx} 41$ that is naturally depleted over the first few days of life. When the levels drop below a certain threshold, the mutants die, demonstrating it is an essential factor. In contrast, zebrafish $d d x 41$ heterozygous animals are phenotypically indistinguishable from wildtype animals during embryogenesis and in adulthood, sug- gesting a $50 \%$ decrease of $\mathrm{Ddx} 41$ alone is insufficient to alter hematopoiesis. This is in agreement with the clinical observation that patients with germline DDX41 mutations who develop hematologic malignancies often acquire somatic missense mutations in the second allele that are thought to diminish DDX41 ATPase activity. Combined, the data indicate that when DDX41 levels decrease to less than $50 \%$, this leads to hematologic defects, but when critically too low, it leads to lethality.

DDX41 was previously identified as a mediator of genomic stability in a cell line-based genome-wide siRNA screen. ${ }^{11}$ However, a role for DDX41 in genomic integrity as well as the downstream consequences of its loss were never demonstrated in vivo. Our current work revealed that $D d \times 41$ regulates genomic integrity in vivo, and that loss of $d d \times 41$ leads to both cell cycle arrest and apoptosis in erythrocytes that contributes to anemia in $d d \times 41$ mutants. We established that ATM and ATR signaling contribute to these attributes, but only ATM inhibition significantly increased o-dianisidine-positive erythrocytes in $d d \times 41$ mutants. The differential impact on oxygenated erythrocyte output by inhibition of ATM and ATR might indicate that Ddx41-regulated ATM signaling is more critical for proper erythropoiesis. ATM has an additional role in apoptosis, especially during development that might explain some of the phenotypic differences when comparing ATM and ATR inhibition effects on erythropoiesis. However, it should be noted that although the ATM and ATR kinases respond uniquely, there exists an extensive 'cross-talk' between them, which can make determining which precise pathway is involved in a phenotype confusing. ${ }^{32}$ Further dissection of the role of DDX41 in ATM and ATR pathway regulation will need to be investigated.

Splicing mutations are commonly found in hematologic malignancies. ${ }^{33,34}$ DDX41 interacts with multiple components of the spliceosome. ${ }^{1}$ Our work aligns with prior studies showing DDX41 insufficiency associates with numerous deleterious splicing outcomes. If and how these splicing events contribute to hematopoietic pathogenesis is unclear. We showed that components related to cell cycle and DNA repair are commonly mis-spliced in $d d \times 41$ mutants. Therefore, it is possible that loss of $d d \times 41$ may be mediating cell cycle arrest and activation of DDR via mis-splicing of crucial regulators of these pathways. The contribution of DDR pathway component mis-splicing in human cytopenias remains to be addressed.

In addition to the effect on cell cycle, we delineated maturation defects in $d d \times 41$ erythrocytes marked by aberrant globin expression and a megaloblastoid-like morphology of mutant erythrocytes. Although we could not perform a complete analysis of definitive erythropoiesis as ddx41 mutants die before EMP-derived or HSC-derived erythrocytes fully mature, the diminished expression of the larval $\beta \mathrm{e} 2$ globin suggests a decrease in EMP-derived definitive erythrocytes. This finding combined with the elevated cmyb and gata1 levels in EMP cells suggests that this defect could be caused in part by maturation defects. As the treatment with the ATM inhibitor KU60019 only partially increased hemoglobinized erythrocytes in $d d \times 41$ mutants, it suggests that deregulation of another pathway underlies additional maturational defects in $d d \times 41$ mutants.

In summary, our study unveils a critical role for $\mathrm{Ddx} 41$ as a key gatekeeper to maintain cell cycle progression, a necessary component for erythrocytic development. We 
A
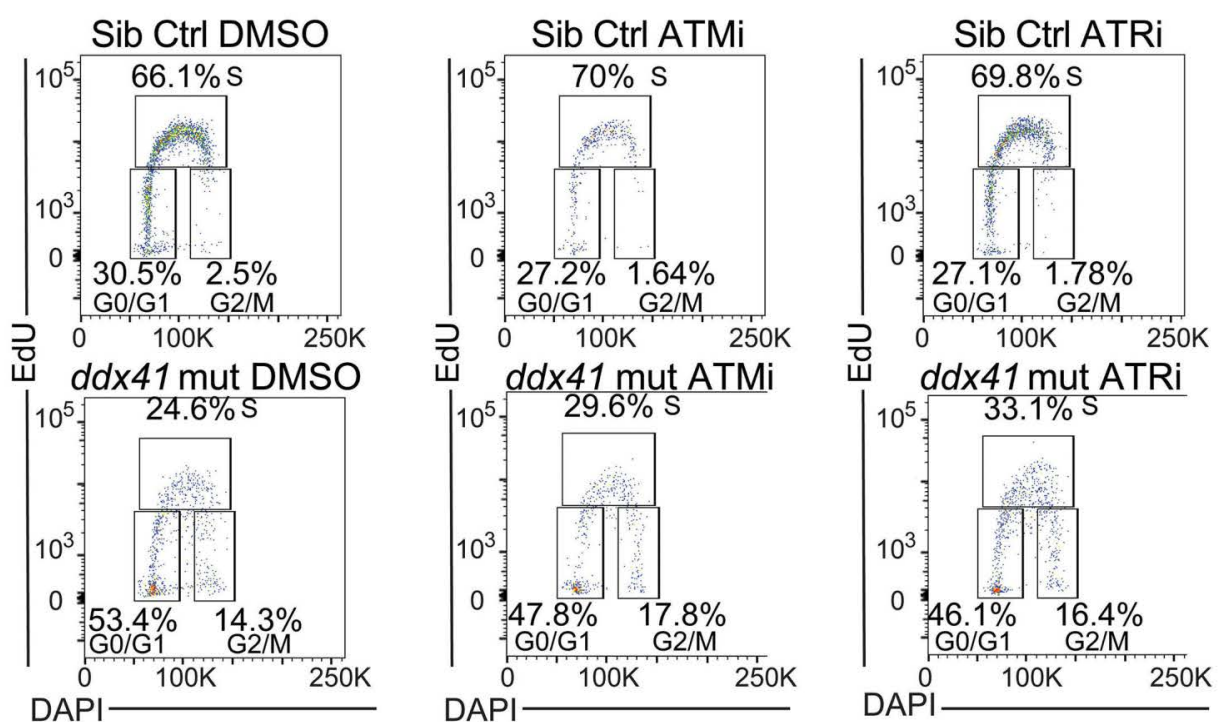

B

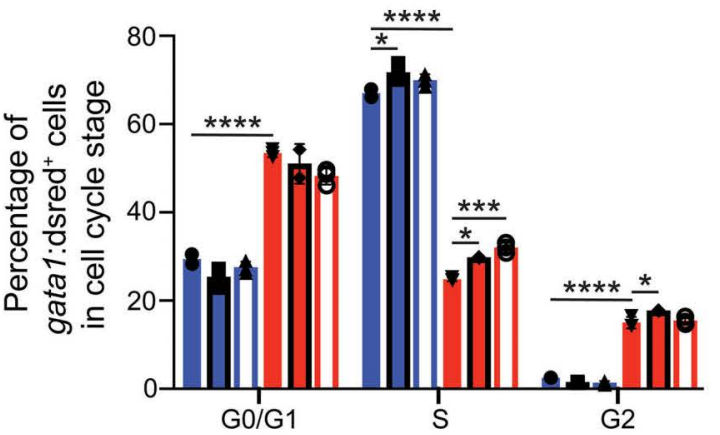

C

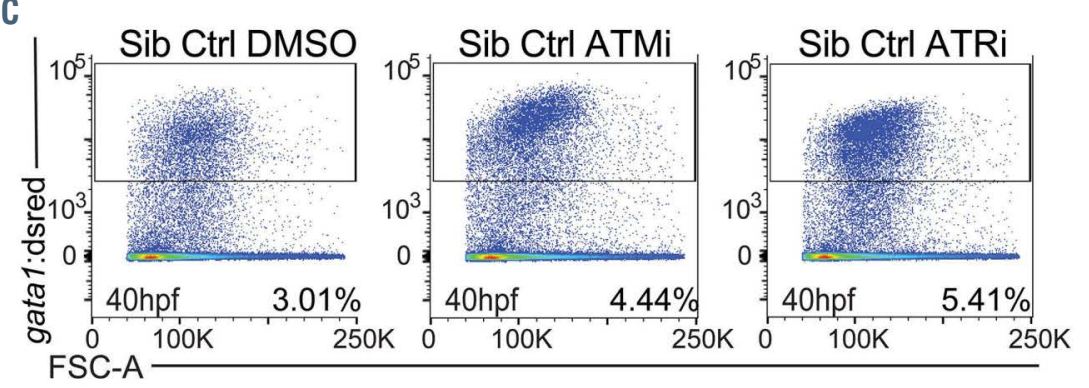

Sib Ctrl DMSO ddx41 mut DMSO

Sib Ctrl ATMi

Sib CtrlATRi

ddx41 mut ATMi

$d d \times 41$ mut ATRi
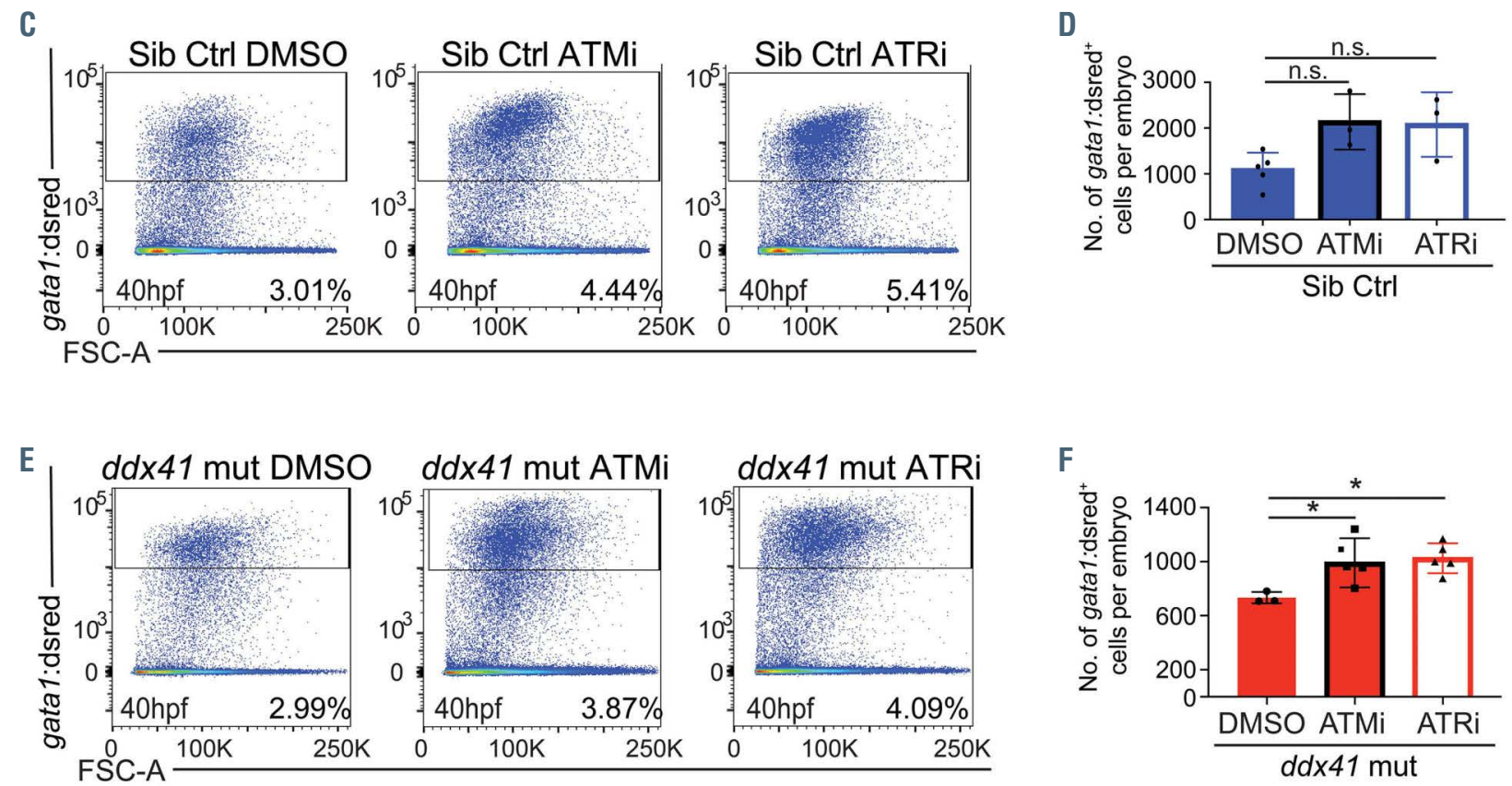

Figure 5. Ddx41 regulation of ATM/ATR signaling contributes to proper erythroid progenitor proliferation. (A) Cell cycle analysis of gata1:dsred ${ }^{+}$erythroid cells from sibling controls (top) and ddx41 mutants (bottom) treated with dimethyl sulfoxide (DMSO) (left), 30 nM KU60019 (Ataxia-telengiectasia-mutated [ATMi] inhibitor, middle), and $30 \mathrm{nM}$ AZ20 (Ataxia-telengiectasia and Rad3-related [ATRi] inhibitor, right) after a 2-hour pulse of 5-ethynyl-2'-deoxyuridine (EdU) at 28 hours post fertilization (hpf). EdU incorporation (y-axis) and DAPI content (x-axis) were measured by flow cytometry at 30 hpf. (B) Quantification of the percentage of cells in each cell cycle phase from (A). (C and E) Flow cytometry plots of gata1:dsred+ erythroid cells from sibling controls (C) and ddx41 mutants (E) treated with DMSO (left), 30 nM KU60019 (ATM inhibitor, middle), and $30 \mathrm{nM} \mathrm{AZ20} \mathrm{(ATR} \mathrm{inhibitor,} \mathrm{right).} \mathrm{(D} \mathrm{and} \mathrm{F).} \mathrm{Graphs} \mathrm{depicting} \mathrm{the} \mathrm{absolute} \mathrm{number} \mathrm{of} \mathrm{gata1:dsred}{ }^{+}$erythroid cells per embryo from (C) and (E). Graphs display means \pm standard deviations (stds) with $P$-values calculated with a one-way ANOVA with Tukey's multiple testing correction, $* P<0.05$, $* * * P \leq 0.001, * * * * P \leq 0.0001$. For flow cytometry, $\mathrm{n}=3-5$ pools of $\sim 5-20$ embryos per pool. 
A

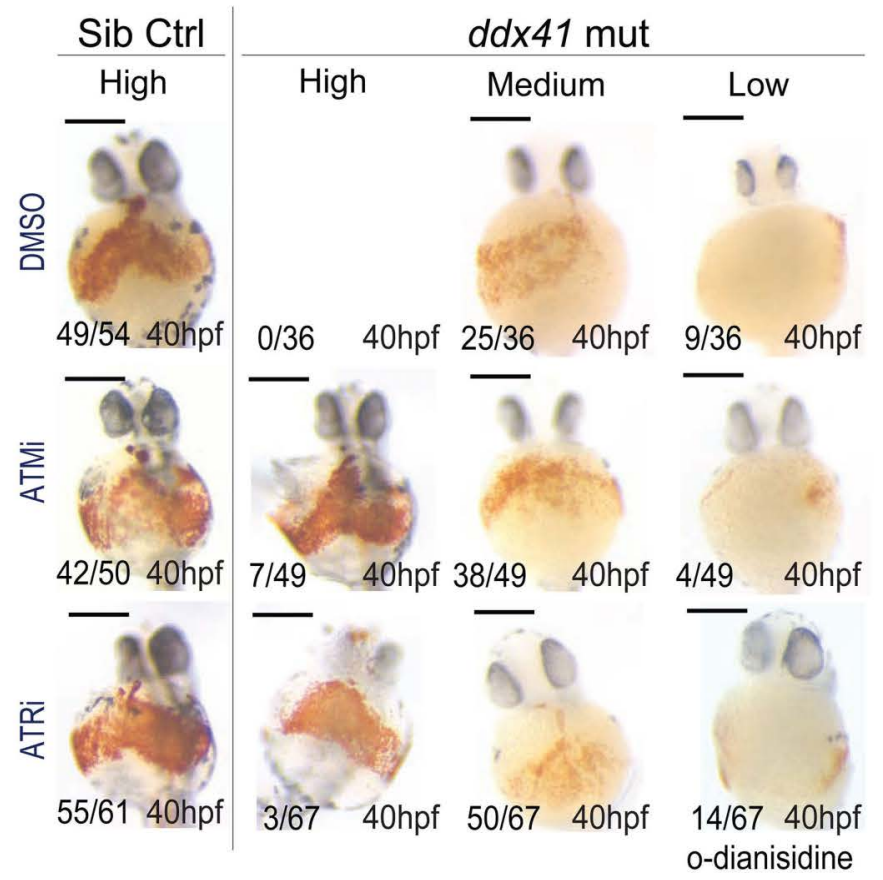

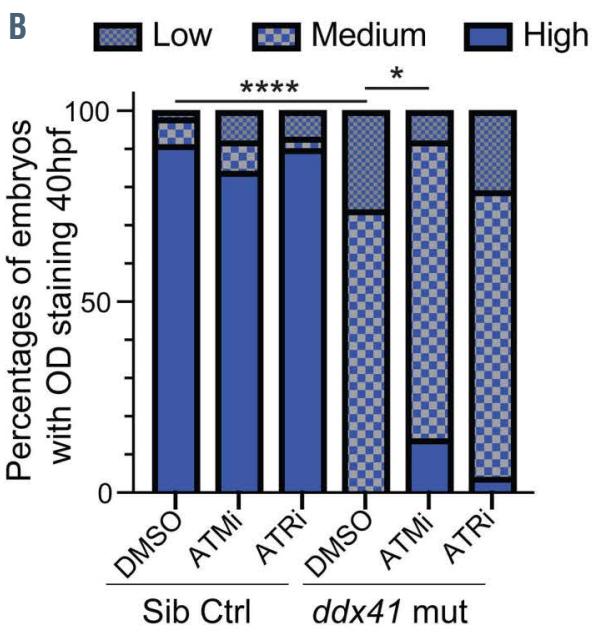

Figure 6. ATM inhibition partially suppresses $d d x 41$ mutant anemia. (A) Representative images of o-dianisidine staining and corresponding levels of staining from sibling controls (left) and ddx41 mutants (right) treated with dimethyl sulfoxide (DMSO) (top), 30 nM KU60019 (Ataxia-telengiectasia-mutated inhibitor [ATMi], middle), and $30 \mathrm{nM}$ AZ20 (Ataxia-telengiectasia and Rad3-related inhibitor[ATRi], bottom). Numbers on bottom left corner indicate the fraction of embryos with the same phenotype as the one depicted in the image. scale bars $=100 \mu \mathrm{m}$. (B) Graph depicting frequency of designated o-dianisidine staining levels in sibling controls and ddx41 mutants at 40 hours post fertilization (hpf) treated with DMSO vehicle control, 30 nM KU60019 (ATMi), and 30 nM AZ20 (ATRi). Graphs display frequency of embryos with each phenotype with $P$-values calculated with Chi-squared test, $* P<0.05, * * * * P \leq 0.0001$. For o-dianisidine staining, $\mathrm{n}=36$ - 67 embryos per experiment.

demonstrated that deficiency of $d d x 41$ triggers cell cycle arrest via activation of ATM and ATR, which ultimately mediates a decrease in proliferation and maturation of erythrocytic progenitors in $d d \times 41$ mutants. These findings establish a critical function for $\mathrm{Ddx} 41$ in promoting healthy erythropoiesis by suppressing genomic stress and present a potential role for ATM and ATR signaling in DDX41-mutant pathologies.

\section{Disclosures}

No conflicts of interest to disclose.

\section{Contributions}

$J T W$ and TVB designed the project experimental approach; $J T W, E S, R W$, and TVB performed the experiments; VG per- formed bioinformatics analysis; JTW and TVB analyzed the data; JTW and TVB wrote and edited the manuscript; all authors reviewed and approved the manuscript.

\section{Acknowledgments}

This work was funded by American Cancer Society RSG129527-DDC, DOD BM180109, NIH 1R01DK121738$01 A 1$ and the Edward P. Evans Foundation (to TVB), NIH MSTP training grant T32GM007288-45 and F30 fellowship 1F30HL142161 (to JTW), and NIH 1R01GM057829-23 to Charles Query for support of VG. We also want to acknowledge the assistance of numerous core facilities at Albert Einstein College of Medicine including Flow Cytometry, Analytical Imaging, and Genomics Facilities (funded by NCI Cancer Grant P30CA013330), and the Zebrafish Core Facility.

\section{References}

1. Polprasert C, Schulze I, Sekeres MA, et al. Inherited and somatic defects in DDX41 in myeloid neoplasms. Cancer Cell. 2015;27(5):658-670.

2. Iacobucci I, Wen J, Meggendorfer M, et al. Genomic subtyping and therapeutic targeting of acute erythroleukemia. Nat Genet. 2019;51(4):694-704.

3. Yoneyama-Hirozane M, Kondo M, Matsumoto SI, et al. High-throughput screening to identify inhibitors of DEAD box helicase DDX41. SLAS Discov. 2017;22(9):1084-1092.

4. Sebert M, Passet M, Raimbault $A$, et al. Germline DDX41 mutations define a significant entity within adult MDS/AML patients. Blood. 2019;134(17):1441-1444.
5. Schneider RK, Schenone M, Ferreira MV, et al. Rps14 haploinsufficiency causes a block in erythroid differentiation mediated by S100A8 and S100A9. Nat Med. 2016;22(3): 288-297.

6. Danilova N, Sakamoto KM, Lin S. Ribosomal protein L11 mutation in zebrafish leads to haematopoietic and metabolic defects. Br J Haematol. 2011;152(2): 217-228.

7. Payne EM, Virgilio M, Narla A, et al. LLeucine improves the anemia and developmental defects associated with DiamondBlackfan anemia and del(5q) MDS by activating the mTOR pathway. Blood. 2012;120(11):2214-2224.

8. Parvatiyar K, Zhang Z, Teles RM, et al. The helicase DDX41 recognizes the bacterial secondary messengers cyclic di-GMP and cyclic di-AMP to activate a type I interferon immune response. Nat Immunol. 2012;13 (12):1155-1161

9. Zhang Z, Yuan B, Bao M, et al. The helicase DDX41 senses intracellular DNA mediated by the adaptor STING in dendritic cells. Nat Immunol. 2011;12(10):959-965.

10. Zhang Z, Bao M, Lu N, et al. The E3 ubiquitin ligase TRIM21 negatively regulates the innate immune response to intracellular double-stranded DNA. Nat Immunol. 2013;14(2):172-178.

11. Paulsen RD, Soni DV, Wollman R, et al. A genome-wide siRNA screen reveals diverse cellular processes and pathways that mediate genome stability. Mol Cell. 2009;35(2):228-239.

12. Lawrence C. Advances in zebrafish husbandry and management. Methods Cell 
Biol. 2011:104:429-451.

13. Kettleborough RN, Busch-Nentwich EM, Harvey SA, et al. A systematic genome-wide analysis of zebrafish protein-coding gene function. Nature. 2013;496(7446):494-497.

14. Traver $\mathrm{D}$, Paw $\mathrm{BH}$, Poss $\mathrm{KD}$, et al. Transplantation and in vivo imaging of multilineage engraftment in zebrafish bloodless mutants. Nat Immunol. 2003;4(12):12381246.

15. De La Garza A, Cameron RC, Nik S, Payne SG, Bowman TV. Spliceosomal component Sf3b1 is essential for hematopoietic differentiation in zebrafish. Exp Hematol. 2016;44 (9):826-837.

16. Thisse C, Thisse B. High-resolution in situ hybridization to whole-mount zebrafish embryos. Nat Protoc. 2008;3(1):59-69.

17. Brownlie A, Hersey C, Oates AC, et al. Characterization of embryonic globin genes of the zebrafish. Dev Biol. 2003;255(1):4861.

18. Liao EC, Paw BH, Oates AC, et al. SCL/Tal1 transcription factor acts downstream of cloche to specify hematopoietic and vascular progenitors in zebrafish. Genes Dev. 1998;12(5):621-626.

19. Detrich HW 3rd, Kieran MW, Chan FY, et al. Intraembryonic hematopoietic cell migration during vertebrate development. Proc Natl Acad Sci U S A. 1995;92(23):1071310717.

20. Dobrzycki T, Krecsmarik M, Bonkhofer F,
Patient R, Monteiro R. An optimised pipeline for parallel image-based quantification of gene expression and genotyping after in situ hybridisation. Biol Open. 2018;7(4):bio031096.

21. Lieschke GJ, Oates AC, Crowhurst $\mathrm{MO}$ Ward AC, Layton JE. Morphologic and functional characterization of granulocytes and macrophages in embryonic and adult zebrafish. Blood. 2001;98(10):3087-3096.

22. Sorrells S, Nik S, Casey $M$, et al. Spliceosomal components protect embryonic neurons from R-loop-mediated DNA damage and apoptosis. Dis Model Mech. 2018;11(2):dmm031583.

23. Carroll KJ, North TE. Oceans of opportunity: exploring vertebrate hematopoiesis in zebrafish. Exp Hematol. 2014;42(8):684 696.

24. Clements WK, Traver D. Signalling pathways that control vertebrate haematopoietic stem cell specification. Nat Rev Immunol. 2013;13(5):336-348

25. Lyons SE, Lawson ND, Lei L, et al. A nonsense mutation in zebrafish gata 1 causes the bloodless phenotype in vlad tepes. Proc Natl Acad Sci U S A. 2002;99(8):5454-5459.

26. Paffett-Lugassy NN, Zon LI. Analysis of hematopoietic development in the zebrafish. Methods Mol Med. 2005;105: 171-198.

27. Bertrand JY, Kim AD, Violette EP, et al. Definitive hematopoiesis initiates through a committed erythromyeloid progenitor in the zebrafish embryo. Development. 2007;134(23):4147-4156.

28. Ganis JJ, Hsia N, Trompouki E, et al Zebrafish globin switching occurs in two developmental stages and is controlled by the LCR. Dev Biol. 2012;366(2):185-194

29. Liberzon A, Birger C, Thorvaldsdottir H, et al. The Molecular Signatures Database (MSigDB) hallmark gene set collection. Cell Syst. 2015;1(6):417-425.

30. Subramanian A, Tamayo P, Mootha VK, et al. Gene set enrichment analysis: a knowledge-based approach for interpreting genome-wide expression profiles. Proc Natl Acad Sci U S A. 2005;102(43):1554515550.

31. Orth JD, Loewer A, Lahav G, Mitchison TJ. Prolonged mitotic arrest triggers partial activation of apoptosis, resulting in DNA damage and p53 induction. Mol Biol Cell. 2012;23(4):567-576

32. Cimprich KA, Cortez D. ATR: an essential regulator of genome integrity. Nat Rev Mol Cell Biol. 2008;9(8):616-627.

33. Yoshida K, Sanada M, Shiraishi Y, et al Frequent pathway mutations of splicing machinery in myelodysplasia Nature. 2011;478(7367):64-69.

34. Haferlach T, Nagata Y, Grossmann V, et al. Landscape of genetic lesions in 944 patients with myelodysplastic syndromes. Leukemia. 2014;28(2):241-247. 